\title{
Radio detection of extensive air showers - Measuring the properties of cosmic rays with the radio technique at LOFAR and the Pierre Auger Observatory
}

\author{
Jörg R. Hörandel ${ }^{\mathrm{a}, \mathrm{b}, \mathrm{c}}$ \\ ${ }^{a}$ Radboud Universiteit, Department of Astrophysics/IMAPP, P.O. Box 9010, 6500 GL Nijmegen, The Netherlands \\ ${ }^{b}$ Nikhef, Science Park 105, 1098 XG Amsterdam, The Netherlands \\ ${ }^{c}$ Vrije Universiteit Brussel, Department of Physics and Astronomy, B-1050 Brussels, Belgium
}

\begin{abstract}
The radio emission from extensive air showers is measured with the LOFAR radio telescope and the Auger Engineering Radio array AERA at the Pierre Auger observatory. The properties of cosmic rays, such as direction, energy and particle type are derived from the radio measurements. Recent results and perspectives for the future are discussed.
\end{abstract}

Keywords: cosmic rays, air showers, radio detection, LOFAR, Pierre Auger Observatory

\section{Introduction}

High-energy cosmic rays impinging on the atmosphere of the Earth initiate cascades of secondary particles, the extensive air showers, schematically illustrated in Fig. 1. Many secondary particles are electrons and positrons. They emit electromagnetic radiation with frequencies of tens of $\mathrm{MHz}$ during the shower development. The bulk of the radiation originates from the transverse separation of charges in the shower due to interactions with the Earth's magnetic field: the geomagnetic effect [1]. One expects the radiation intensity being proportional to $v \times B$, where $v$ denotes the direction of the particles in the shower (shower axis) and $B$ the Earth magnetic field. The geomagnetic radiation is linearly polarized.

A second, sub-dominant component arises through the longitudinal separation of charges in the shower (Askaryan effect [6]). This component is radially polarized. The direction of the electric field within the shower is measured with high resolution with LOFAR [7]. This allows a quantitative evaluation of the contribution of the two emission processes to the overall

Email address: horandel@astro.ru.nl (Jörg R. Hörandel) URL: particle.astro.ru.nl (Jörg R. Hörandel)

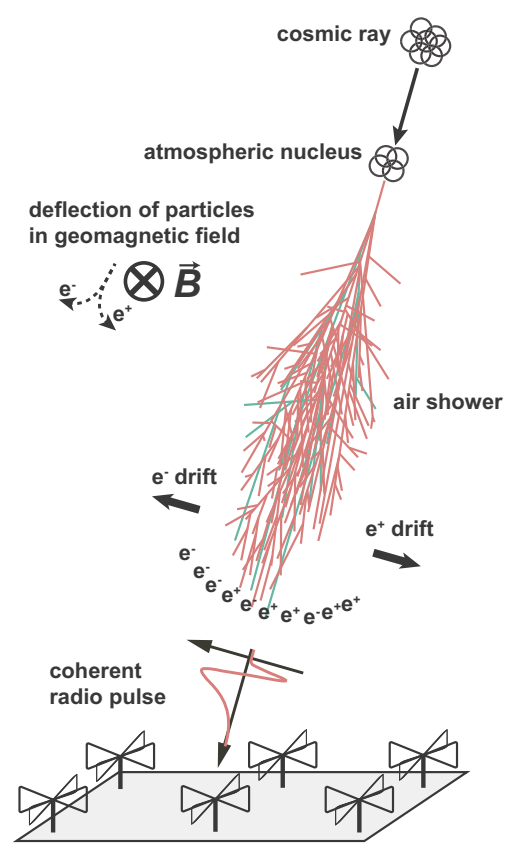

Figure 1: Schematic view of an air shower developing in the atmosphere. Electromagnetic radiation with frequencies of tens of $\mathrm{MHz}$ is emitted through the transverse and longitudinal separation of charges in the shower. 

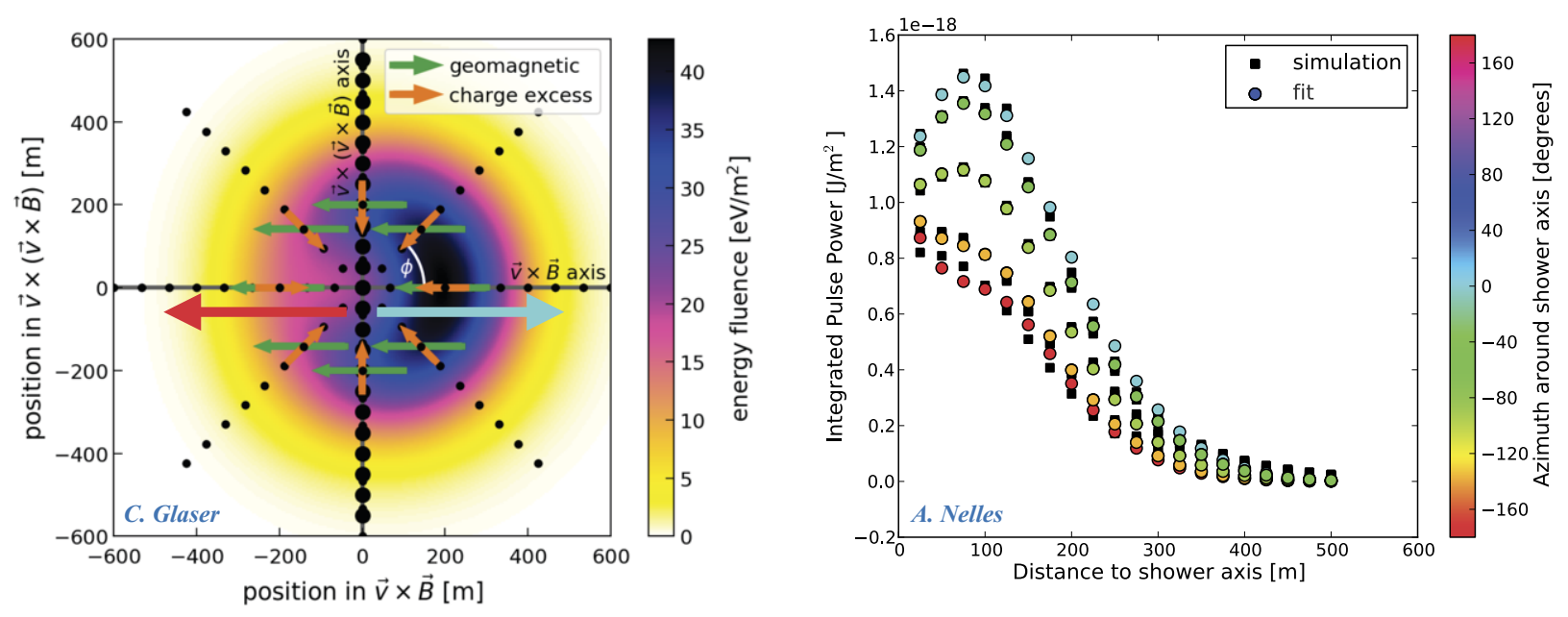

Figure 2: Left: Distribution of the energy fluence (in the $30-80 \mathrm{MHz}$ band) of an air shower with $60^{\circ}$ zenith angle [2]. Superimposed is the polarization direction of the geomagnetic and charge-excess emission processes at different positions in form of arrows. Simulated with CORSIKA/CoREAS [3, 4]. Right: Lateral distribution of the radiation power on the ground for bins in azimuthal angle, following a parameterization described in [5].

shower emission. The results yield a dependence on the zenith angle and the distance to the shower axis for the ratio of the two radiation components. Values for the ratio range from about $5 \%$ to $20 \%$, with an average contribution of the Askaryan effect of about $15 \%$. A similar values has been obtained through polarization measurements with AERA [8].

Interference between the two radiation components with their different polarization behavior leads to a nonrotationally symmetric distribution of the radiation intensity on the ground, as illustrated in Fig. 2 (left). This directly implies a non-trivial behavior of the lateral density distribution of the radio emission. Using the dense LOFAR core $[5,9]$ it became obvious that the lateral density distribution is not rotationally symmetric and a simple description as e.g. proposed in [10] is not sufficient. Instead now, more complex, two-dimensional functions are being used to describe the azimuthal dependence of the radiation strength $[5,2]$. As an example, a parameterization introduced in [5] is depicted in Fig. 2 (right). The colors represent different intervals in azimuthal angles. The non-rotational symmetry is clearly visible. The big arrows on the left-hand panel of Fig. 2 indicate two azimuthal angles of $0^{\circ}$ and $180^{\circ}$, respectively.

\section{LOFAR and AERA}

We focus here on the description of recent results obtained by the LOFAR radio telescope and the Auger Engineering Radio Array (AERA) at the Pierre Auger ob- servatory. The layout of both installations is illustrated to scale in Fig. 3.

LOFAR $[11,12]$ is a distributed radio telescope. Its antennas are distributed over northern Europe with the densest concentration in the north of the Netherlands. The antennas of LOFAR are grouped into stations. A station consist of 96 low-band antennas $(10-90 \mathrm{MHz})$ and 48 high-band antennas $(110-240 \mathrm{MHz})$. Both frequency bands are used for the observation of air showers $[9,13]$. The 23 stations within the $\sim 5 \mathrm{~km}^{2}$ core are distributed in an irregular pattern that maximizes uv-coverage, or spatial frequencies for standard interferometric observations. At the center of the LOFAR core six stations are located in a roughly $320 \mathrm{~m}$ diameter area, called the Superterp, providing both the shortest baselines for interferometric observations and the densest population of antennas ideal for cosmic-ray observations. An array of scintillation detectors installed on the Superterp complements the set-up $[14,15]$. The particle detector array is used to measure the properties of air showers and to issue a trigger signal to read out the radio antennas.

The AERA project $[16,17]$ comprises more than 150 antennas installed on an area covering about $17 \mathrm{~km}^{2}$ inside the Auger observatory in the field of view of the High Elevation Telescopes (HEAT). HEAT are fluorescence light detectors aimed to measure air showers with energies above $10^{17} \mathrm{eV}$. The radio antennas are installed on sub-grids with different spacings, ranging from $144 \mathrm{~m}$ to $375 \mathrm{~m}$ in order to compensate for the change of the size of the radio footprint as a function of 


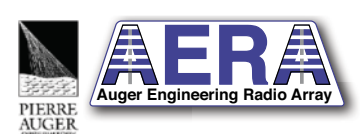

$\sim 150$ antennas $\sim 17 \mathrm{~km}^{2}$ 30-80 MHz
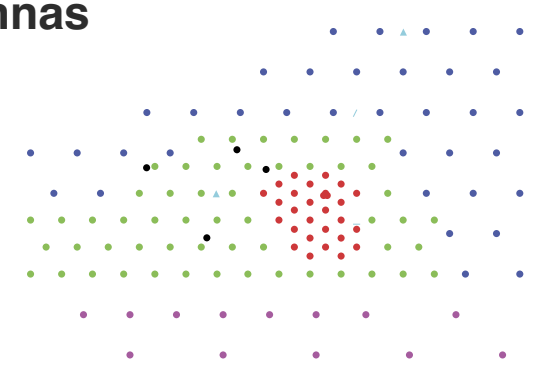

- $\quad$ - $\quad$ -
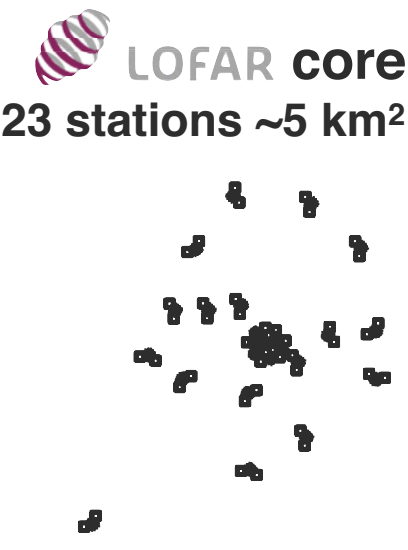

$>2000$ antennas

Figure 3: Layout of the radio antennas from AERA at the Pierre Auger Observatory (left) and the core of the LOFAR radio telescope (right), drawn to scale.

cosmic-ray energy. Two types of antennas are installed [18]: 24 stations are equipped with two logarithmicperiodic dipole antennas, oriented in magnetic NorthSouth and East-West directions. The signals are amplified and filtered before they are introduced into a filteramplifier and digitization chain. There, they are band pass filtered between 30 and $80 \mathrm{MHz}$ and digitized. The remaining antennas are an active bowtie antenna also called "butterfly". This antenna is highly sensitive towards the ground which enhances the antenna gain. All Radio Detector stations operate autonomously employing solar power systems.

Both installations are calibrated in situ with high precision, both for the signal strength $[19,20]$ and the signal arrival time [21, 22].

\section{Properties of cosmic rays}

The ultimate goal of the radio measurements of air showers is to derive the properties of the incoming cosmic rays, namely their arrival direction, energy and particle type (nuclear mass).

The direction of the cosmic ray is obtained through a precise measurement of the arrival time of the wavefront at the individual antennas of an array. LOFAR, with its high antenna density was valuable to clearly understand the precise shape of the radio wavefront in air showers [23] and it could be shown that a hyperboloid is an
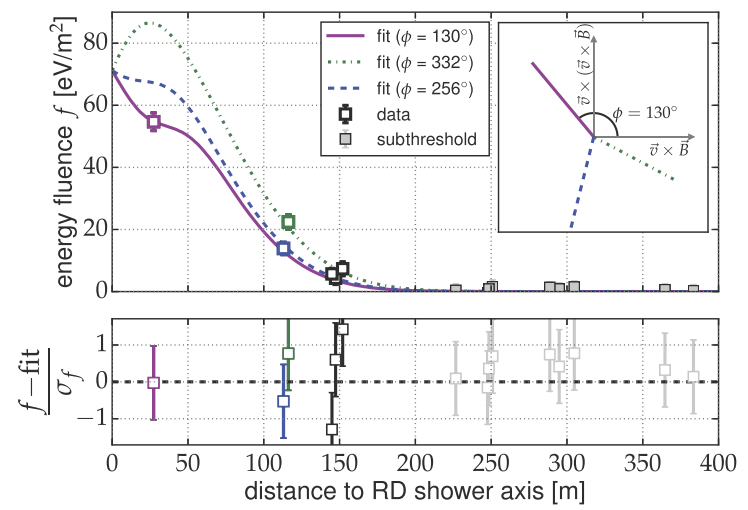

Figure 4: Energy fluence of an air shower with an energy of 4.4 . $10^{17} \mathrm{eV}$ and a zenith angle of $25^{\circ}$ as measured in individual AERA radio detectors $[25,26]$.

appropriate analytical description. This confirms earlier work by LOPES [24]. From measurements of the arrival time of the shower front the direction of the cosmic rays can be determined with a resolution of better than $1^{\circ}$.

The energy of the air shower is derived from the measurement of the radio energy density on the ground. The energy fluence of a measured air shower is depicted in Fig. $4[25,26]$. A function [5] is fitted to the measured values in order to calculate the integral of the total energy in the frequency band $30-80 \mathrm{MHz}$ transported in an air shower to the ground. This quantity is propor- 


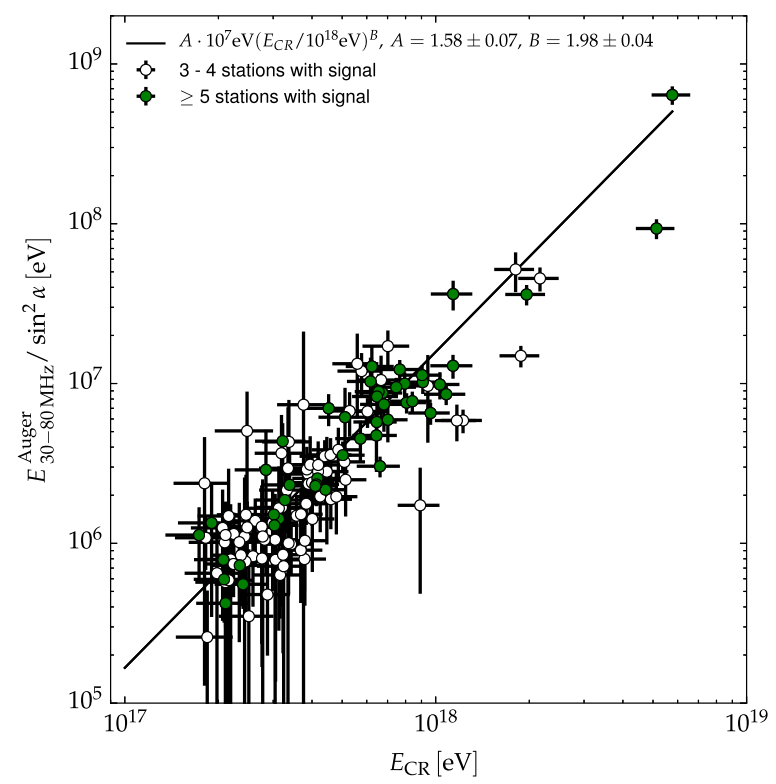

Figure 5: Correlation between the normalized radio energy and the cosmic-ray energy as determined by the Auger Surface Detector. Open circles represent air showers with radio signals detected in three or four radio detectors. Solid circles denote showers with five or more detected radio signals $[25,26]$.

tional to the shower energy as illustrated in Fig. 5 where the normalized radio energy is plotted as a function of the cosmic-ray energy as measured with the Auger Surface Detector. For a set of high-quality showers an energy resolution of better than $25 \%$ has been achieved $[26,25]$.

At LOFAR, using results from simulations and a comparison to measurements from a particle detector array an energy resolution around $30 \%$ is obtained [9, 27].

A cosmic ray with an energy of $1 \mathrm{EeV}$ delivers about $15.8 \mathrm{MeV}$ of energy to the ground in the frequency range from 30 to $80 \mathrm{MHz}[26,25]$. The radio emission measured on the ground $E_{30-80 \mathrm{MHz}}$ can be used to establish an absolute calibration of the eneryg scale $E_{\mathrm{CR}}$, using the universal formula

$$
\begin{aligned}
E_{30-80 \mathrm{MHz}}=[15.8 & \pm 0.7 \text { (stat) } \pm 6.7 \text { (syst) }] \mathrm{MeV} \\
& \times\left(\sin \alpha \frac{E_{\mathrm{CR}}}{10^{18} \mathrm{eV}} \frac{B_{\text {Earth }}}{0.24 \mathrm{G}}\right)^{2},
\end{aligned}
$$

knowing the magnetic field $B_{\text {Earth }}$ at the location of the detector and the angle $\alpha$ between the direction of the Earth magnetic field and the direction of the air shower.

Experimentally, the most challenging subject is the determination of the particle type or its nuclear mass. The basic observable in air shower physics is the depth of the shower maximum $X_{\max }$, being proportional to the nuclear mass $A$ of the cosmic ray $X_{\max } \propto \ln A$. The

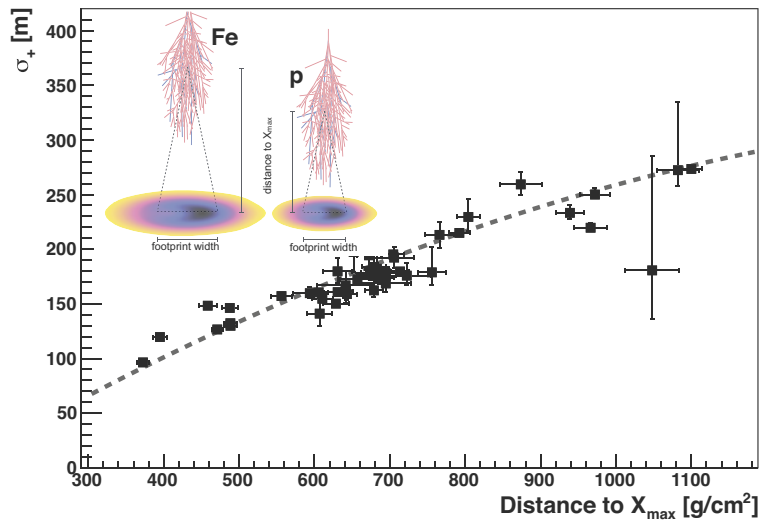

Figure 6: Size of the air shower footprint as a function of the distance from the antennas to the shower maximum as obtained with CORSIKA/CoREAS simulations [9]. The inset illustrates the underlying principle for air showers induced by a proton and an iron nucleus.

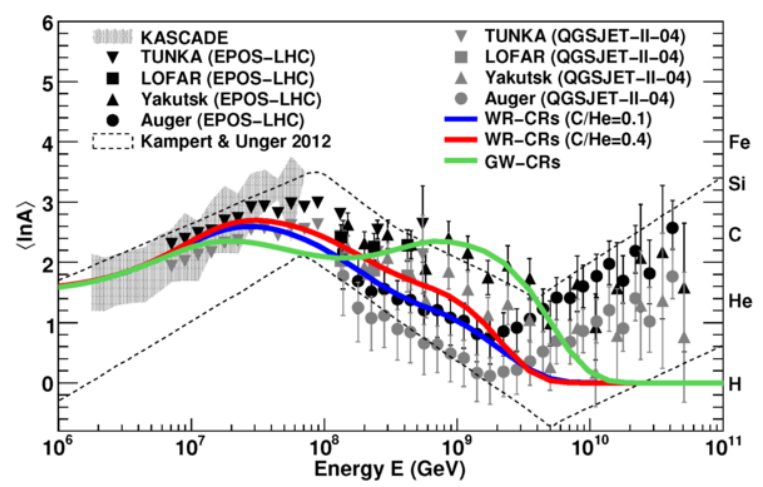

Figure 7: Mass composition of cosmic rays. The mean logarithmic mas is plotted as a function of the energy [28].

radio signals are in principle emitted on the Čerenkov cone. Thus, the size of the footprint of the radio emission on the ground scales well with the distance from the emission region (basically $X_{\max }$ ) to the antennas (on the ground) [9]. This is depicted in Fig. 6. Knowing the vertical profile of the atmosphere, the depth of the shower maximum $X_{\max }$ can be derived. For LOFAR a highprecision method has been developed to measure $X_{\max }$ $[29,30]$. For each measured cosmic ray dedicated simulations are conducted, taking into account the measured energy and direction of the cosmic ray. The predicted signals in the particle and radio detectors are compared to the measured values on a statistical basis and a best fit value is obtained for $X_{\max }$. A resolution fo $X_{\max }$ of better than $20 \mathrm{~g} / \mathrm{cm}^{2}$ has been obtained. 


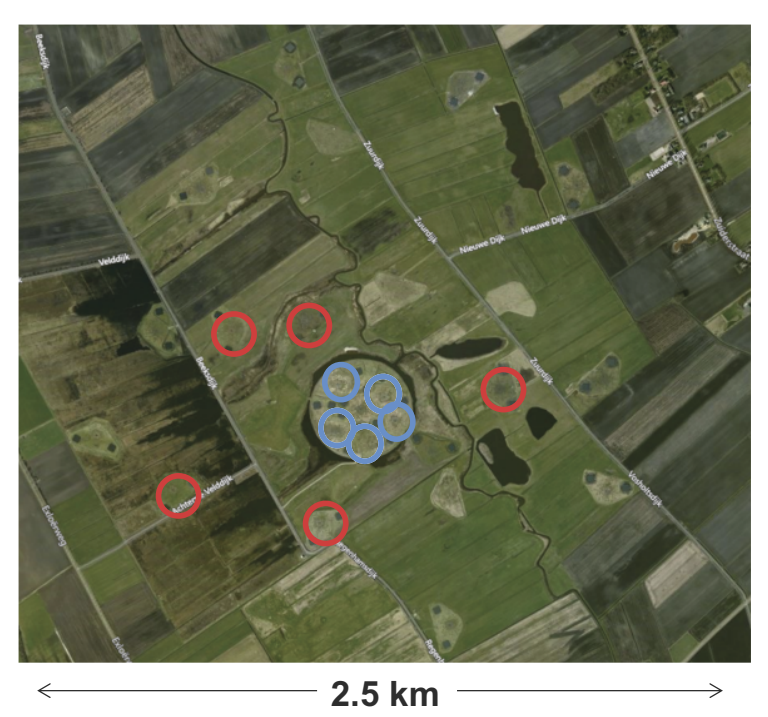

Existing station

New station

Figure 8: Satellite image of the LOFAR dense core, illustrating the extension of the scintillator array. Blue circles indicate existing stations with particle detectors. In addition, particle detectors will be installed at the stations indicated with red circles.

The mean logarithmic mass of cosmic rays

$$
\ln A=\frac{X_{\max }^{\text {meas }}-X_{\max }^{F e}}{X_{\max }^{p}-X_{\max }^{F e}}
$$

has been calculated from the measured $X_{\max }$ values and predictions from simulations for the depth of the shower maximum for protons $X_{\max }^{p}$ and iron nculei $X_{\max }^{F e}$. The result is presented in Fig. 7 as a function of cosmic-ray energy together with the world data [28]. As can be inferred from the figure, the data derived with the radio technique are well compatible with the world data in the energy region between $10^{17}$ and $10^{18} \mathrm{eV}$, where a transition from a Galactic to an extra-galactic origin of cosmic rays is expected.

This method is also applied to AERA data. The obtained $X_{\max }$ values from the radio measurements have been compared to $X_{\max }$ observations from the fluorescence light telescopes. In first analyses a resolution of the order of $40 \mathrm{~g} / \mathrm{cm}^{2}$ has been achieved. The resolution depends on the number of antennas participating in a measurement and thus, on the quality selection cuts applied [31].

Combining the various efforts around the world one can state that the radio detection technique is now mature and the properties of cosmic rays are now being measured on a regular basis with such devices with

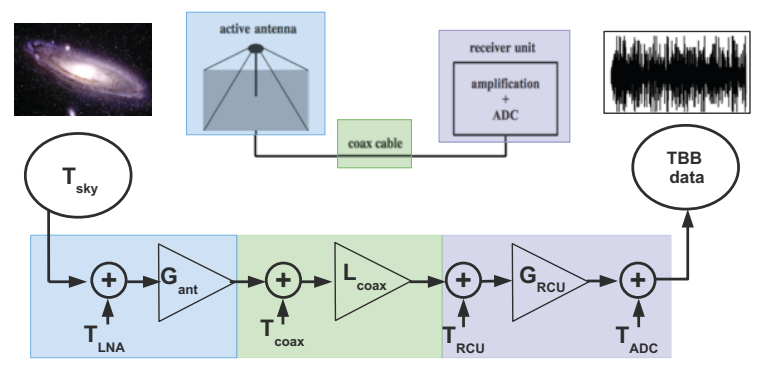

Figure 9: LOFAR signal chain, illustrating the various steps from the observed signal on the sky to recorded ADC values, stored in ring buffers (transient buffer board - TBB) [33].

state-of-the-art accuracies of the order of $0.1^{\circ}$ to $0.5^{\circ}$ for the arrival direction, $20 \%$ to $30 \%$ for the energy, and 20 to $40 \mathrm{~g} / \mathrm{cm}^{2}$ for the depth of the shower maximum (particle type), with the accuracies mainly depending on the antenna density of the respective installation.

\section{Ongoing work and prospects for the future}

\subsection{LOFAR}

At present the particle detector array at the LOFAR observatory is being extended. Five LOFAR stations around the Superterp are being equipped with scintillator stations, as illustrated in Fig. 8. At each station four scintillator detectors, $1 \mathrm{~m}^{2}$ each, identical to the ones described in [14] will be installed. This will increase the effective area of the LORA particle detector array. At energies between $10^{16}$ and $10^{18} \mathrm{eV} \mathrm{a} \approx 45 \%$ increase of the number of air showers with a radio signal is expected [32].

At present the calibration procedure for LOFAR is also being improved [33], further reducing the systematic uncertainties with respect to previous work [19]. Radio emission from the Galactic center is used as a reference source. The complete signal chain, end to end, from the signal on the sky to the recorded ADC traces is evaluated, as illustrated in Fig. 9. This method requires knowing the contributions of electronic noise to the system, and the original Galactic calibration is limited by the systematic uncertainty of the electronic noise. This is being improved through the ongoing efforts by modeling each step in the signal chain in order to understand the electronic noise introduced into the system. Using this method, a new absolute and frequency dependent calibration has been derived with a maximum of $14 \%$ systematic uncertainty for frequencies below $77 \mathrm{MHz}$. A comparison of the logarithmic slopes of calibrated, measured power spectra and simulated power spectra shows promising agreement. 


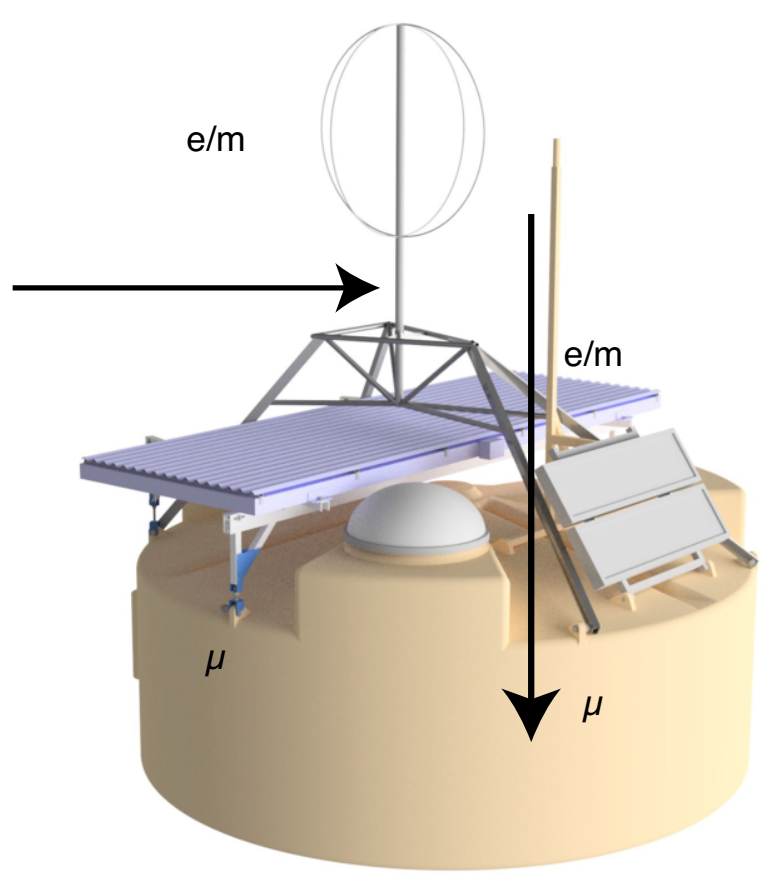

Figure 10: Conceptional illustration of an upgraded station of the Surface Detector array of the Pierre Auger observatory, comprised of (from bottom to top) a water-Čerenkov detector, a layer of scintillators, and a radio antenna [34]. The arrows indicate the detection principles for vertical and horizontal air showers. In practice, the electromagnetic and muonic components are obtained by unfolding the signals from the Surface Scintillator Detector and the water-Čerenkov detector

\subsection{The radio upgrade of the Pierre Auger observatory}

At present, the Pierre Auger collaboration is working on an upgrade of the Observatory [35]. The physics case of the upgrade is outlined in [36]. The key science questions to be addressed are: What are the sources and acceleration mechanisms of ultra-high-energy cosmic rays (UHECRs)? Do we understand particle acceleration and physics at energies well beyond the LHC (Large Hadron Collider) scale? What is the fraction of protons, photons, and neutrinos in cosmic rays at the highest energies?

It is planned to install a radio antenna on each of the 1661 stations of the Surface Detector array of the observatory, forming a $3000 \mathrm{~km}^{2}$ radio array, the largest radio array for cosmic-ray detection in the world [34, 37]. An artists impression of the planned set-up is given in Fig. 10. It shows (from bottom to top) the water-Čerenkov detector with a layer of scintillators on top (Surface Scintillator Detector) and a radio antenna. With the combination of water-Čerenkov detector and Surface Scintillator Detector the electron-to-muon ratio $(\mathrm{e} / \mu)$ is measured for vertical showers. In a similar way the

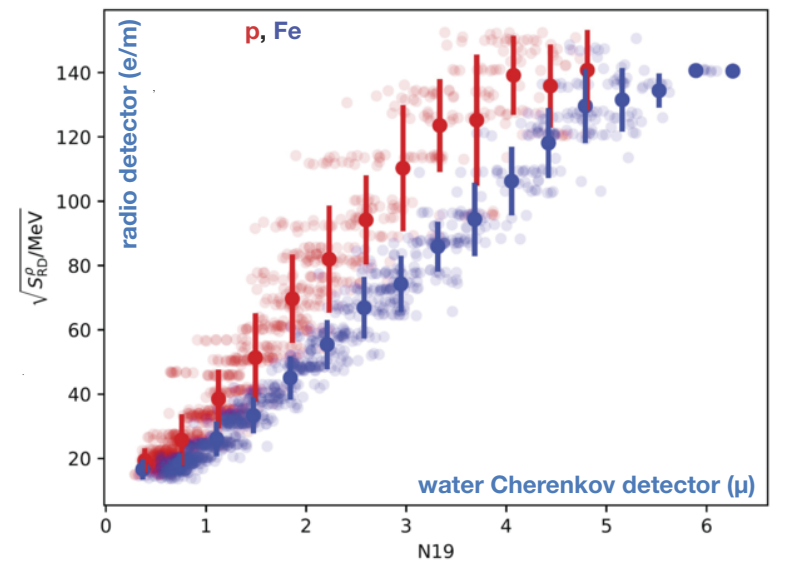

Figure 11: Electromagnetic energy in a shower as measured by the Radio Detector as a function of the muon content in a shower as measured by the water-Čerenkov detector [34]. Preliminary results from simulations are shown.

combination of water-Čerenkov detector and Radio Detector will be used to measure the $\mathrm{e} / \mu$ ratio for horizontal air showers. In turn, the $\mathrm{e} / \mu$ ratio will be used to derive the particle type of the incoming cosmic ray up to the highest energies. This is the main goal of the Auger upgrade, to measure the particle type of each incoming cosmic ray. Thus, the radio upgrade will increase the aperture of the observatory for mass-sensitive investigations, enabling $\mathrm{e} / \mu$ separation for showers with a broad zenith angle range, from the zenith with the Surface Scintillator Detector to the horizon with the Radio Detector.

There is an important difference related to the mass measurement of cosmic rays with AERA (for vertical showers) and the radio upgrade (for horizontal air showers). For vertical showers we use a geometrical method, correlating the size of the footprint on the ground to the distance from the observer to the shower maximum $[16,38,27]$. From this quantity, the depth of the shower maximum $X_{\max }$ is derived, which in turn is dependent on the logarithm of the number of nucleons (nuclear mass) $A$ of the incoming cosmic ray $X_{\max } \propto \ln A$ [16]. For horizontal showers we aim to apply a different method: We will use a combination of radio antennas and the water-Čerenkov detectors to measure the $\mathrm{e} / \mu$ ratio in air showers to determine $\ln A$.

In a first analysis the physics potential of the radio upgrade has been studied using CORSIKA[3]/CoREAS[4] simulations. 192 showers have been simulated, induced by half protons, have iron nuclei, with energies from 4 to $40 \mathrm{EeV}$ and zenith angles between $60^{\circ}$ to $80^{\circ}$. A full water-Čerenkov detector detector simulation and re- 


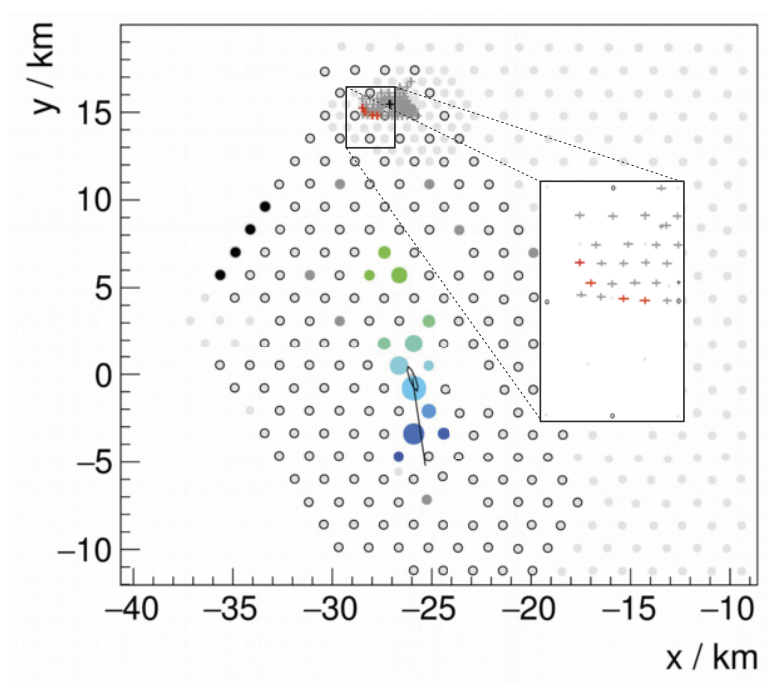

Figure 12: A measured horizontal air shower. The blue to green circles indicate the measurement with the Surface Detector, size indicating energy deposit and color encoding arrival time. Dark-grey circles indicate isolated particle detections rejected in the reconstruction. The radio signal extends over a significantly larger area than the particle distribution [40].

construction has been included in the analysis, but only a simplified treatment of the radio signals was available. The normalized radiation energy has been calculated as described in [39]. The radiation energy has been "smeared out" in order to mimic reconstruction uncertainties. Preliminary results are illustrated in Fig. 11. The size of the e/m component (as measured with the Radio Detector) is plotted as a function of the muon number (as obtained with the water-Čerenkov detector). A clear separation between showers induced by protons and iron nuclei is visible. Please note: the flattening at the top-right is due to a technical flaw in the simulations, only showers up to a certain (too low) energy have been simulated.

The concept to measure horizontal air showers with an antenna array with km-scale spacing has been verified with the existing AERA at the Auger observatory [40, 41]. An interesting air shower is shown in Fig. 12. The air shower has been detected with four antennas at the edge of AERA. Its readout was triggered because an isolated Surface Detector station with significant energy deposit (dark-grey circles in Fig. 12) was closer than the $5 \mathrm{~km}$ maximum readout distance, presently set in the AERA data acquisition. The locations of the antennas with a signal are in alignment with the ground projection of the air-shower axis reconstructed from the Surface Detector data. The azimuth angles reconstructed from the radio signals and particle-detector measure-

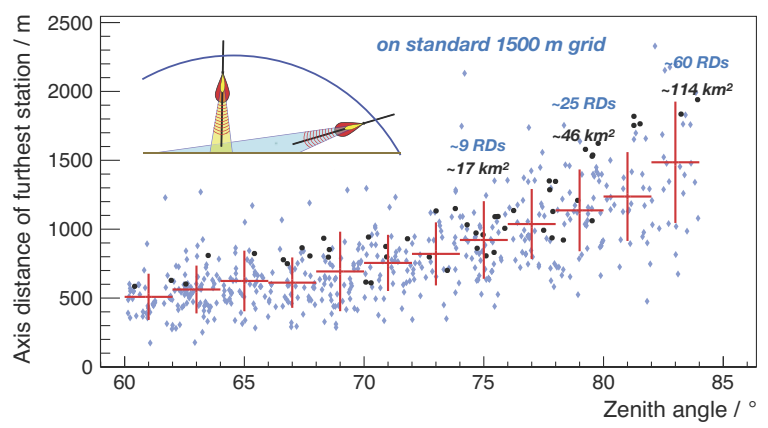

Figure 13: Furthest axis distance at which a radio signal above noise background has been detected with AERA as a function of the airshower zenith angle [40].

ments agree to within better than $0.5^{\circ}$. The zenith angle reconstructed with the particle detectors amounts to $83^{\circ}$, while the zenith angle determined from the arrival times of the radio signals corresponds to $87^{\circ}$. The low number of radio antennas with signal and their approximate alignment along a line perpendicular to the air-shower axis limit the zenith-angle resolution of the radio measurement in this particular case. The maximum axial distance at which a signal has been measured amounts to $2150 \mathrm{~m}$ and the exceptionally large ground distance of the measured radio signals, more than $15 \mathrm{~km}$ away from the shower axis arises from projection effects. Nevertheless, this example illustrates that the ground area illuminated by radio signals can be significantly larger than the particle footprint on the ground.

The size of the radio footprint of an air shower has been measured. The distance from the shower axis to the furthest away radio detector (in the shower plane, perpendicular to the shower axis) is depicted in Fig. 13 as a function of the zenith angle of the air shower. In the figure also the expected number of radio stations (on the standard Auger $1500 \mathrm{~m}$ grid) with a signal above threshold are indicated together with an estimate of the size of the footprint on the ground. As can be seen, for horizontal air showers (i.e. large zenith angles) the footprints reach sizes exceeding tens of $\mathrm{km}^{2}$ and dozens of stations will have a signal above threshold. This is a very important result since it confirms experimentally that the radio emission from horizontal air showers can bee measured with radio antennas on the standard Auger $1500 \mathrm{~m}$ grid.

[1] F. Kahn, I. Lerche, Radiation from cosmic ray air showers, Royal Society of London Proceedings Series A 289 (1966) 206.

[2] C. Glaser, S. de Jong, M. Erdmann, J. R. Hörandel, An analytic description of the radio emission of air showers based on its emission mechanisms, Astropart. Phys. 104 (2019) 64-77. arXiv:1806.03620, doi:10.1016/j.astropartphys.2018.08.004. 
[3] D. Heck, et al., Corsika: A monte carlo code to simulate extensive air showers, Report FZKA 6019, Forschungszentrum Karlsruhe (1998).

[4] T. Huege, M. Ludwig, C. W. James, Simulating radio emission from air showers with coreas, ARENA 2012, AIP Conf. Proc. 1535 (2013) 128-132doi:http://dx.doi.org/10.1063/1.4807534.

[5] A. Nelles, et al., A parameterization for the radio emission of air showers as predicted by CoREAS simulations and applied to LOFAR measurements, Astropart.Phys. 60 (2015) 13-24. arXiv:1402.2872, doi:10.1016/j.astropartphys.2014.05.001.

[6] G. Askaryan, Excess negative charge of the electron-photon shower and coherent radiation originating from it. radio recording of showers under the ground and on the moon,, Journal of the Physical Society of Japan Supplement 17 (1962) C257.

[7] P. Schellart, et al., Polarized radio emission from extensive air showers measured with LOFAR, JCAP 1410 (10) (2014) 014. arXiv:1406.1355, doi:10.1088/1475-7516/2014/10/014.

[8] A. Aab, et al., Probing the radio emission from air showers with polarization measurements, Phys.Rev. D89 (5) (2014) 052002. arXiv:1402.3677, doi:10.1103/PhysRevD.89.052002.

[9] A. Nelles, et al., The radio emission pattern of air showers as measured with LOFAR - a tool for the reconstruction of the energy and the shower maximum, JCAP 1505 (05) (2015) 018. arXiv:1411.7868, doi:10.1088/1475-7516/2015/05/018.

[10] H. Allan, Progress in Elementary Particles and Cosmic Ray Physics, J.G. Wilson and S.G. Wouthuysen eds., North Holland, 1971, p. 169.

[11] M. van Haarlem, et al., LOFAR: The LOw-Frequency ARray, Astronomy \& Astrophysics 556 (2013) A2. arXiv:1305.3550.

[12] P. Schellart, et al., Detecting cosmic rays with the LOFAR radio telescope, Astron.Astrophys. 560 (2013) A98. arXiv:1311.1399, doi:10.1051/0004-6361/201322683.

[13] A. Nelles, et al., Measuring a Cherenkov ring in the radio emission from air showers at $110-190 \mathrm{MHz}$ with LOFAR, Astropart.Phys. 65 (2014) 11-21. arXiv:1411.6865, doi:10.1016/j.astropartphys.2014.11.006.

[14] S. Thoudam, et al., LORA: A scintillator array for LOFAR to measure extensive air showers, Nucl.Instrum.Meth. A767 (2014) 339-346. arXiv:1408.4469, doi:10.1016/j.nima.2014.08.021.

[15] S. Thoudam, et al., Measurement of the cosmic-ray energy spectrum above $10^{16} \mathrm{eV}$ with the LOFAR Radboud Air Shower Array, Astropart. Phys. 73 (2016) 34-43. arXiv:1506.09134, doi:10.1016/j.astropartphys.2015.06.005.

[16] J. Schulz, et al., Pierre Auger Collaboration, Status and prospects of the auger engineering radio array, Proceedings 34th International Cosmic Ray Conference, Den Haag (2015) PoS(ICRC2015)615.

[17] P. Abreu, et al., Advanced functionality for radio analysis in the Offline software framework of the Pierre Auger Observatory, Nucl.Instrum.Meth. A635 (2011) 92-102. arXiv:1101.4473, doi:10.1016/j.nima.2011.01.049.

[18] P. Abreu, et al., Antennas for the Detection of Radio Emission Pulses from Cosmic-Ray, JINST 7 (2012) P10011. arXiv:1209.3840, doi:10.1088/1748-0221/7/10/P10011.

[19] A. Nelles, et al., Calibrating the absolute amplitude scale for air showers measured at LOFAR, JINST 10 (11) (2015) P11005. arXiv:1507.08932, doi:10.1088/1748-0221/10/11/P11005.

[20] A. Aab, et al., Calibration of the logarithmic-periodic dipole antenna (LPDA) radio stations at the Pierre Auger Observatory using an octocopter, JINST 12 (10) (2017) T10005. arXiv:1702.01392, doi:10.1088/1748-0221/12/10/T10005.

[21] A. Corstanje, et al., Timing calibration and spectral cleaning of LOFAR time series data, Astron. Astrophys. 590 (2016) A41. arXiv:1603.08354, doi:10.1051/0004-6361/201527809.
[22] A. Aab, et al., Nanosecond-level time synchronization of autonomous radio detector stations for extensive air showers, JINST 11 (01) (2016) P01018. arXiv:1512.02216, doi: $10.1088 / 1748-0221 / 11 / 01 / \mathrm{P} 01018$.

[23] A. Corstanje, et al., The shape of the radio wavefront of extensive air showers as measured with LOFAR, Astropart.Phys. 61 (2015) 22-31. arXiv:1404.3907, doi:10.1016/j.astropartphys.2014.06.001.

[24] W. Apel, et al., The wavefront of the radio signal emitted by cosmic ray air showers, JCAP 1409 (09) (2014) 025. arXiv:1404.3283, doi:10.1088/1475-7516/2014/09/025.

[25] A. Aab, et al., Measurement of the Radiation Energy in the Radio Signal of Extensive Air Showers as a Universal Estimator of Cosmic-Ray Energy, Phys. Rev. Lett. 116 (24) (2016) 241101. arXiv:1605.02564, doi:10.1103/PhysRevLett.116.241101.

[26] A. Aab, et al., Energy Estimation of Cosmic Rays with the Engineering Radio Array of the Pierre Auger Observatory, Phys. Rev. D93 (12) (2016) 122005. arXiv:1508.04267, doi:10.1103/PhysRevD.93.122005.

[27] S. Buitink, et al., Method for high precision reconstruction of air shower $X_{\max }$ using two-dimensional radio intensity profiles, Phys.Rev. D90 (8) (2014) 082003. arXiv:1408.7001, doi:10.1103/PhysRevD.90.082003.

[28] S. Thoudam, J. P. Rachen, A. van Vliet, A. Achterberg, S. Buitink, H. Falcke, J. R. Hörandel, Cosmic-ray energy spectrum and composition up to the ankle - the case for a second Galactic component, Astronomy \& Astrophysics 595 (2016) 33. arXiv: 1605.03111.

[29] S. Buitink, et al., Measuring the cosmic ray mass composition with LOFAR, Proceedings 34th International Cosmic Ray Conference, Den Haag (2015) PoS(ICRC2015)368.

[30] S. Buitink, et al., A large light-mass component of cosmic rays at $10^{17}-10^{17.5} \mathrm{eV}$ from radio observations, Nature 531 (2016) 70. arXiv:1603.01594, doi:10.1038/nature16976.

[31] F. Canfora, et al., Cosmic ray composition measurements with the auger engineering radio array, Proceedings ARENA 2018.

[32] S. Buitink, et al., Measurement of cosmic rays with lofar, Proceedings ARENA 2018.

[33] K. Mulrey, et al., Updated calibration of the lofar low-band antennas, Proceedings ARENA 2018.

[34] J. Hörandel, A large radio array at the pierre auger observatory, Proceedings ARENA 2018.

[35] A. Castellina, et al., Augerprime: the pierre auger observatory upgrade, Proceedings UHECR symposium, Paris.

[36] A. Aab, et al., The Pierre Auger Observatory Upgrade - Preliminary Design Report, arXiv (2016) 1604.03637arXiv:1604.03637.

[37] J. Hörandel, et al., Precision measurements of cosmic rays up to the highest energies with a large radio array at the pierre auger observatory, Proceedings UHECR symposium, Paris.

[38] T. Huege, et al., Radio detection of cosmic rays with the auger engineering radio array, Proceedings UHECR symposium, Paris.

[39] C. Glaser, M. Erdmann, J. R. Hörandel, T. Huege, J. Schulz, Simulation of Radiation Energy Release in Air Showers, JCAP 1609 (09) (2016) 024. arXiv:1606.01641, doi:10.1088/1475$7516 / 2016 / 09 / 024$.

[40] A. Aab, et al., Observation of inclined EeV air showers with the radio detector of the Pierre Auger Observatory, JCAP 1810 (10) (2018) 026. arXiv:1806.05386, doi:10.1088/1475$7516 / 2018 / 10 / 026$.

[41] M. Gottowik, et al., Measurements of horizontal air showers with the auger engineering radio array, Proceedings ARENA 2018. 